Iller, Carola; Wienberg, Jana

\title{
Altern und Geschlecht. Gesundheit und Wohlbefinden im Alter in einer geschlechterdifferenziellen Perspektive
}

Moser, Vera [Hrsg.]; Rendtorff, Barbara [Hrsg.]: Riskante Leben? Geschlechterordnungen in der Reflexiven Moderne. Opladen u.a. : Verlag Barbara Budrich 2012, S. 83-92. - (Jahrbuch Frauen- und Geschlechterforschung in der Erziehungswissenschaft; 8)

Quellenangabe/ Reference:

Iller, Carola; Wienberg, Jana: Altern und Geschlecht. Gesundheit und Wohlbefinden im Alter in einer geschlechterdifferenziellen Perspektive - In: Moser, Vera [Hrsg.]; Rendtorff, Barbara [Hrsg.]: Riskante Leben? Geschlechterordnungen in der Reflexiven Moderne. Opladen u.a. : Verlag Barbara Budrich 2012, S. 83-92 - URN: urn:nbn:de:0111-pedocs-99367 - DOI: 10.25656/01:9936

https://nbn-resolving.org/urn:nbn:de:0111-pedocs-99367

https://doi.org/10.25656/01:9936

in Kooperation mit / in cooperation with:

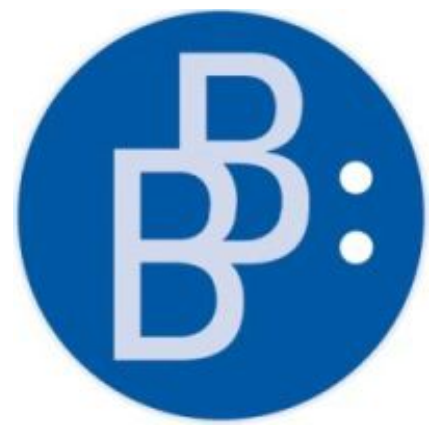

https://www.budrich.de

\section{Nutzungsbedingungen}

Gewährt wird ein nicht exklusives, nicht übertragbares, persönliches und beschränktes Recht auf Nutzung dieses Dokuments. Dieses Dokument ist ausschließlich für den persönlichen, nicht-kommerziellen Gebrauch bestimmt. Die Nutzung stellt keine Übertragung des Eigentumsrechts an diesem Dokument dar und gilt vorbehaltlich der folgenden Einschränkungen Auf sämtlichen Kopien dieses Dokuments müssen alle Urheberrechtshinweise und sonstigen Hinweise auf gesetzlichen Schutz beibehalten werden. Sie dürfen dieses Dokument nicht in irgendeiner Weise abändern, noch dürfen Sie dieses Dokument für öffentliche oder kommerzielle Zwecke vervielfältigen, öffentlich ausstellen, aufführen, vertreiben oder anderweitig nutzen.

Mit der Verwendung dieses Dokuments erkennen Sie die Nutzungsbedingungen an.

\section{Terms of use}

We grant a non-exclusive, non-transferable, individual and limited right to using this document.

This document is solely intended for your personal, non-commercial use. Use of this document does not include any transfer of property rights and it is conditional to the following limitations: All of the copies of this documents must retain all copyright information and other information regarding legal protection. You are not allowed to alter this document in any way, to copy it for public or commercial purposes, to exhibit the document in public, to perform, distribute or otherwise use the document in public.

By using this particular document, you accept the above-stated conditions of use.

\section{Kontakt / Contact:}

peDOCS

DIPF | Leibniz-Institut für Bildungsforschung und Bildungsinformation Informationszentrum (IZ) Bildung

E-Mail: pedocs@dipf.de

Internet: www.pedocs.de

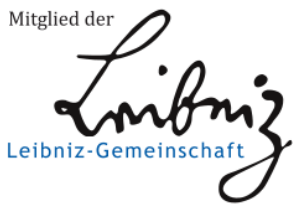


Riskante Leben? Geschlechterordnungen in der Reflexiven Moderne 
Jahrbuch

Frauen- und Geschlechterforschung

in der Erziehungswissenschaft

herausgegeben von

Sabine Andresen

Rita Casale

Edgar Forster

Edith Glaser

Vera Moser

Annedore Prengel

Barbara Rendtorff

Beirat

Birgit Althans, Trier

Eva Borst, Mainz

Eva Breitenbach, Bochum

Bettina Dausien, Wien

Isabell Diehm, Bielefeld

Hannelore Faulstich-Wieland, Hamburg

Carola Iller, Heidelberg

Marita Kampshoff, Schwäbisch Gmünd

Margret Kraul, Göttingen

Andrea Liesner, Hamburg

Susanne Maurer, Marburg

Astrid Messerschmidt, Karlsruhe

Inga Pinhard, Frankfurt

Folge 8/2012 
Vera Moser

Barbara Rendtorff (Hrsg.)

Riskante Leben?

Geschlechterordnungen in der Reflexiven Moderne

Verlag Barbara Budrich

Opladen, Berlin \& Toronto 2012 
Bibliografische Information der Deutschen Nationalbibliothek

Die Deutsche Nationalbibliothek verzeichnet diese Publikation in der Deutschen

Nationalbibliografie; detaillierte bibliografische Daten sind im Internet über

http://dnb.d-nb.de abrufbar.

Gedruckt auf säurefreiem und alterungsbeständigem Papier.

Alle Rechte vorbehalten.

C 2012 Verlag Barbara Budrich, Opladen, Berlin \& Toronto

www.budrich-verlag.de

\section{ISBN 978-3-86649-468-8}

Das Werk einschließlich aller seiner Teile ist urheberrechtlich geschützt. Jede Verwertung außerhalb der engen Grenzen des Urheberrechtsgesetzes ist ohne Zustimmung des Verlages unzulässig und strafbar. Das gilt insbesondere für Vervielfältigungen, Übersetzungen, Mikroverfilmungen und die Einspeicherung und Verarbeitung in elektronischen Systemen.

Satz: Susanne Albrecht-Rosenkranz, Leverkusen

Umschlaggestaltung: disegno visuelle kommunikation, Wuppertal - www.disenjo.de Druck: paper \& tinta, Warschau

Printed in Europe 
Inhalt

\section{Einleitung}

Vera Moser/Barbara Rendtorff

Riskante Leben? Geschlechterordnungen in der Reflexiven Moderne ....

\section{Einführender Essay}

Hildegard Maria Nickel

Individualisierung und Subjektivierung aus der Geschlechterperspektive:

Riskante Chancen

\section{Hauptbeiträge}

Christine Thon

Individualisierte Geschlechterordnungen? Feministische und hegemonieanalytische Kritik eines modernisierungstheoretischen Konzepts

Barbara Pichler

Die flexible Frau und der gebrochene Mann. Geschlechterformationen im sozialpädagogischen Alter(n)sdiskurs

Michèle Amacker

„Und seit dann bin ich einfach daheim, Modell Hausmann.“ Prekäre

Konstellationen: Lebensführung von Haushalten in prekären Lebenslagen zwischen Erwerbs- und Care-Arbeit"

\section{Aus der Forschung}

Carola Iller/Jana Wienberg

Altern und Geschlecht - Gesundheit und Wohlbefinden im Alter in einer geschlechterdifferenziellen Perspektive

Regina Heimann

Bildungsentscheidungen zwischen milieubedingtem Aufstiegswunsch und geschlechtsbezogener Traditionalisierung 
Sandra Glammeier

„Sonst eine ganz toughe Frau“. Erwartete und verhinderte

Selbstbestimmung von Frauen - Paradoxien im Kontext von

Geschlechterkonstruktionen im Modernisierungsprozess

Michael Ley

Geschlechterordnung als institutionelle Abwehr. Untersuchungen zur

Einführung der Koedukation an einer katholischen Mädchenschule

Marion Ott/Rhea Seehaus

„Es ist halt durchs Stillen, dadurch ergibt es sich einfach.“ Familiale

Arbeitsteilungsmuster und Naturalisierungseffekte von Stilldiskursen

John Litau/Barbara Stauber

Riskante Identitätsarbeit? Zur Herstellung von Männlichkeit und

Weiblichkeit in jugendkulturellem Rauschtrinken

\section{Tagungsberichte}

Anna Eggers

Bericht zur ersten Jahrestagung der Fachgesellschaft

Geschlechterstudien/Gender Studies Association ,Verletzbarkeiten.

Geschlechterwissenschaftliche Perspektiven"

Selma Haupt

Bericht zur Jahrestagung der Sektion Frauen- und Geschlechterforschung

in der DGfE an der Universität Paderborn 2011

\section{Rezensionen}

Selma Haupt

Rezension zu: Power: Die eindimensionale Frau

Vera Moser

Rezension zu: Haker: Hauptsache gesund? Ethische Fragen der

Pränatal- und Präimplantationsdiagnostik

Edith Glaser

Rezension zu: Hoffmann: Weibliche Arbeitswelten in der

Wissenschaft. Frauen an der Preußischen Akademie der

Wissenschaften zu Berlin 1890-1945

Claudia Nikodem

Rezension zu: Rendtorff: Bildung der Geschlechter

Sigrid Schmitz

Rezension zu: Fine: Delusions of Gender. The Real Science Behind

Sex Differences 
Markus Gippert

Rezension zu: Mergl: Der Terror der Selbstverständlichkeit.

Widerstand und Utopien im Neo-Individualliberalismus

Sabine Toppe

Rezension zu: Dackweiler/Schäfer (Hrsg.): Wohlfahrtstaatlichkeit

und Geschlechterverhältnisse aus feministischer Perspektive

AutorInnenhinweise

197

Ankündigung

201 


\title{
Altern und Geschlecht - Gesundheit und Wohlbefinden im Alter in einer geschlechterdifferenziellen Perspektive
}

\author{
Carola Iller und Jana Wienberg
}

\begin{abstract}
Frauen und Männer altern nicht in gleicher Weise. Die Gründe für Unterschiede in den Alternsprozessen sind allerdings nicht in einer unterschiedlichen biologischen Ausstattung, sondern im Zusammenspiel von genetischer Disposition und Lebensstil zu suchen. In dem Beitrag wird deshalb der Frage nachgegangen, ob bzw. inwieweit in der zweiten Lebenshälfte Geschlechtsunterschiede in der Lebensführung, in der Zeit- und Aktivitätsstruktur, der Beurteilung des subjektiven Wohlbefindens sowie hinsichtlich der Partizipation an Bildung erkennbar sind und damit Unterschiede in den Lebenslagen, insbesondere im Gesundheitszustand im Alter, erklärt werden können. Die Analyse stiutzt sich auf eine Metaanalyse nationaler sowie internationaler Studienergebnisse zu Bildung und Gesundheit im Alter mit dem Blick auf (vermeintliche) Geschlechtsunterschiede.
\end{abstract}

\section{Ageing and gender - gender differential in health and wellbeing of the elderly}

Men and women go through different ageing processes. However, this difference is not explained by biological factors, but by a complex interaction of lifestyle choices and genetic disposition. This article addresses to what extent way of life, structure of activities, time-scheduling, perceived wellbeing and participation in educational activities differ between men and women in the second half of their life. The analysis is based on a meta-analysis of national and international studies addressing gender specific education and health during the ageing process. 


\section{Einleitung}

Vor dem Hintergrund des demografischen Wandels sind das Interesse an und die Anzahl von Untersuchungen zur Lebenssituation und insbesondere zu Einflussfaktoren auf die Lebenszufriedenheit und Gesundheit im Alter immens gestiegen. Bislang wurden Geschlechterunterschiede hierbei allerdings nur am Rande thematisiert, wobei sich insgesamt recht widersprüchliche Erkenntnisse zu männlichen und weiblichen Lebenslagen im Alter gegenüber stehen. Einerseits wird angenommen, dass Geschlechterrollen im Alter ihre normative Kraft verlieren und es zu einer partiellen Annäherung (vgl. Sørenson 1990) der Entwicklungsverläufe sowie einer Angleichung der Lebensentwürfe von Frauen und Männern im Alter kommt (Mayer 1991). Andererseits ist festzustellen, dass sich auch im höheren Alter die Lebenssituation, die sozioökonomischen Bedingungen, Aktivitätsmuster und Partizipationsmöglichkeiten zwischen den Geschlechtern unterscheiden. Dies betrifft insbesondere den Gesundheitszustand, der gerade im höheren Alter deutliche Unterschiede in Abhängigkeit von Dauer und Intensität der Belastungen im Lebensverlauf aufweist. Dies gilt vor allem für belastende Arbeitsbedingungen, wie Schichtarbeit, dauerhafte Verausgabung in beruflichen und privaten Anforderungssituationen, aber auch lebensstilabhängige Verhaltensweisen wie einseitige Ernährung und Bewegungsarmut, die erst über einen längeren Zeitraum von mehreren Jahren wirksam werden. Die Lebensqualität im Alter wird also nicht nur von aktuellen Gegebenheiten, sondern maßgeblich von biografisch erworbenen Ressourcen und nachwirkenden Belastungen bestimmt, so zeigen übereinstimmend empirische Ergebnisse der Alternsforschung (vgl. Iller 2005; Kuhlmey et al. 2007; Kickbusch 2006). Eine besondere Rolle spielt dabei Bildung, denn ihr kann hinsichtlich der Gesundheit im Alter eine protektive Funktion zugesprochen werden (vgl. zusammenfassend zum Forschungsstand Wienberg 2010): Bildung ermöglicht es gesundheitlichen Beeinträchtigungen im Alter vorzubeugen bzw. vorhandene Beeinträchtigungen zu kompensieren, sie kann als Aktivität zu gesellschaftlicher Teilhabe im Alter beitragen und den Reflexionsraum der eigenen Lebensgestaltung erweitern.

Angesichts der komplexen Wirkmechanismen wäre es deshalb zu kurz gegriffen, Unterschiede oder Annäherungen in den Lebenslagen von Frauen und Männern im Alter vorrangig auf die genetische Disposition zurückzuführen. Unsere These ist vielmehr, dass Geschlechterunterschiede im Alter in den Lebenslagen, insbesondere hinsichtlich der Gesundheit, bestehen bleiben, sofern die über den Lebensverlauf angebahnten Unterschiede in der Lebensweise fortbestehen. Eine Verschiebung in der Disparität setzt eine reflexive Auseinandersetzung insbesondere mit den geschlechterdifferenten Aktivitätsmustern, sozialer Unterstützung und der Kompetenz zur Gestaltung eines zufriedenen und selbstbestimmten Lebens voraus. 
Wie zu zeigen sein wird, sind Frauen und Männer unterschiedlich von Beeinträchtigungen im Alter betroffen. Wir konzentrieren uns bei der folgenden Analyse auf Gesundheit im Alter, wobei wir Gesundheit im Sinne der Weltgesundheitsorganisation (WHO) als ,Zustand des vollständigen körperlichen, geistigen und sozialen Wohlbefindens" (s. Verfassung der Weltgesundheitsorganisation von 1946) verstehen. Gesundheit soll insofern nicht, zumindest nicht ausschließlich, als ein objektiver, biometrisch messbarer Tatbestand angesehen werden, sondern als Kategorie des individuellen Erlebens von Wohlbefinden (vgl. Tesch-Römer/Wurm 2009: 12). Gesundheit ist in diesem Verständnis ein entscheidender Gradmesser zur Bewertung von Teilhabe und Selbstbestimmung.

Auf der Grundlage einer Metaanalyse von nationalen sowie internationalen empirischen Studien zu Gesundheit im Alter ${ }^{1}$ werden wir zunächst die Befundlage zu (vermeintlichen) Geschlechterunterschieden im Gesundheitszustand im Alter vorstellen. Im zweiten Schritt werden wir dann mögliche Erklärungen diskutieren. Im dritten und letzten Abschnitt werden wir uns mit Perspektiven und Fragestellungen für weitere Forschung befassen.

\section{Befunde zu Unterschieden im Gesundheitszustand von Frauen und Männern im Alter}

Altern zeichnet sich durch eine große Multidimensionalität, Multidirektionalität und Multikausalität aus, d.h. sie kann in ihrer temporären Entwicklung wie auch im Ausmaß sehr unterschiedlich verlaufen (Schmitt et al. 2008: $12 \mathrm{f}$.). In den meisten gerontologischen Studien werden deshalb soziodemografische Angaben erhoben, um Testergebnisse entsprechend einordnen zu können. Die meisten uns bekannten Studien weisen auch Geschlechterverteilungen in den Befunden aus. Allerdings wurden bisher keine systematischen geschlechterdifferenziellen Auswertungen vorgenommen, die auf die unterschiedliche Lebenssituation von Männern und Frauen im Alter Bezug nehmen. Im Folgenden können die Befunde zum Gesundheitszustand deshalb nur deskriptiv nach Geschlechtszugehörigkeit der StudienteilnehmerInnen dargestellt werden. Rückschlüsse auf Geschlechtsunterschiede in der Lebenssituation und in Lebensverläufen sind auf dieser Basis nicht möglich.

Aufgrund der bislang feststellbaren Übersterblichkeit der Männer - u.a. wegen demografischer Bedingungen, wie kriegsbedingte Ausfälle in den

1 Bei der Studienauswahl wurden ausschließlich medizinisch angelegte Untersuchungen nicht berücksichtigt, da sie zu dem hier interessierenden soziodemografischen Kontext keine Informationen liefern. Das Design und die Datengrundlage der Metaanalyse sind ausführlich beschrieben in Wienberg 2010. 
männlichen Kohorten und die höhere Lebenserwartung ${ }^{2}$ von Frauen - ist eine Ungleichheit in der Geschlechterverteilung zwischen alten Menschen zu konstatieren, die von Tews als „Feminisierung des Alters" bezeichnet wird (Tews 1993: 28ff.; Tews 1999: 148f.). Die Geschlechtsunterschiede in der Lebenserwartung sind möglicherweise darauf zurückführen, dass bestimmte Krankheiten häufiger und intensiver bei Männern als bei Frauen auftreten. Allerdings bestehen bzgl. der Mortalitätsursache keine Unterschiede zwischen den Geschlechtern: Die mit Abstand häufigsten Todesursachen sind Herzerkrankungen und Neubildungen (vgl. Statistisches Bundesamt 2009).

Obgleich Frauen eine höhere Lebenserwartung aufweisen, sind hinsichtlich der körperlich-organischen Gesundheit allerdings keine generellen Geschlechterunterschiede im hohen Alter festzustellen. Betrachtet man die statistischen Zahlen zur Multimorbidität, so schneiden Frauen allerdings signifikant schlechter ab, d.h. Frauen leiden mit zunehmendem Alter häufiger als Männer unter multiplen chronischen Krankheiten (Lindenberger et al. 2010: 626). Des Weiteren wird ein Geschlechtsunterschied in Hinblick auf die Gedächtnisleistungen konstatiert: Einer schwedischen Studie zufolge haben Frauen im Vergleich zu Männern ein besseres episodisches Gedächtnis (vgl. Betula - Aging, Memory and Dementia 2005). In der deutschen ILSE-Studie wurde eine leichte kognitive Beeinträchtigung mit vergleichbarer Häufigkeit beider Geschlechter identifiziert, Alzheimer-Demenz war bei Männern häufiger (Männer 61,5\% vs. Frauen 38,5\%) (Schröder et al. 2008: 36). Zusammenfassend zeigen sich folgende geschlechtsbezogenen Differenzierungen bzgl. der Morbidität und Mortalität:

- Insgesamt sind Frauen - insbesondere im Alter von unter 25 Jahren und über 65 Jahren - öfter von Krankheit betroffen, in den Altersgruppen dazwischen sind es überwiegend Männer (vgl. Statistisches Bundesamt 2009). Die Prävalenz für psychiatrische Erkrankungen (mit Ausnahme Schizophrenie) und allgemein für Morbidität ist bei Frauen höher als bei Männern. Zudem sind Frauen von neurotischen und psychosomatischen Störungen doppelt so häufig betroffen wie Männer. Chronisch-degenerative Erkrankungen (Erkrankungen des Muskel-Skelett-Systems und Herz-Kreislauferkrankungen) treten bei Frauen häufiger auf(Clemens 1997: 97f.).

- Insgesamt sind mehrheitlich Frauen in ärztlicher Behandlung, Diagnosen chronischer Erkrankungen sind bei ihnen häufiger (Statistisches Bundesamt 2005: 71f.).

2 Statistisch besteht in Deutschland derzeit (noch) eine Differenz der Lebenserwartung der Geschlechter von durchschnittlich fünf bis sieben Jahren. Die durchschnittliche Lebenserwartung liegt aktuell bei 77,8 (Männer) und 82,7 (Frauen) Jahren (vgl. Statistisches Bundesamt 2008a, 2008b). Vermutlich wird zukünftig absolut und relativ am stärksten die $\mathrm{Be}$ setzungszahl der Männer - trotz höherer Sterblichkeit - in den allerhöchsten Altersstufen zunehmen, da es keine kriegsbedingten Unterbesetzungen der Männerjahrgänge geben wird (Baltes et al. 1994: 83). 
- Laut Todesursachenstatistik überwiegt die Mortalitätsrate bei Frauen hinsichtlich Herz-Kreislauf-Krankheiten deutlich gegenüber den Männern, bei Neubildungen und Krankheiten der Atemorgane liegen Frauen statistisch hinter den Männern (Statistisches Bundesamt 2005: 16f.).

Im Hinblick auf die subjektive Beurteilung des eigenen Gesundheitszustandes ist laut den Ergebnissen der EdAge-Studie die Zufriedenheit bei den Männern höher, die Gesundheitszufriedenheit sinkt bei den Männern jedoch rapider als bei den Frauen (Tippelt/Schmidt 2009: 95). Zu ähnlichen Befunden kam auch die Berliner Altersstudie (2010), wonach die Frauen ihren Gesundheitszustand tendenziell schlechter beurteilen als Männer (Lindenberger et al. 2010: 608), sowie die in der Schweiz durchgeführte Interdisziplinäre Altersstudie (IDA), der zufolge Frauen zwar generell gesundheitsbewusster sind, jedoch ihre Gesundheit zugleich pessimistischer beurteilen und zudem vermehrt über physische Beschwerden berichten (vgl. Perrig-Chiello et al. 1999).

Zahlreiche Untersuchungen weisen darauf hin, dass der Gesundheitszustand im Alter stark von sozialen Ressourcen (vgl. Weber/Glück et al. 2005) und insbesondere vom Bildungsstand abhängig ist (vgl. zusammenfassend Wienberg 2010; Iller/Wienberg 2010). In diesem Zusammenhang ist festzustellen, dass keine Geschlechterunterschiede im Gesundheitszustand erkennbar sind, wenn Frauen und Männer annähernd die gleichen Bildungsabschlüsse und sozioökonomischen Voraussetzungen aufweisen (vgl. WHO MONICA Projekt 2009). Dieses Ergebnis stützt unsere These, dass Geschlechterunterschiede in den Lebenslagen im Alter vorrangig auf Unterschiede in der Lebensweise zurückzuführen sind.

Ausgehend von unserer Annahme, dass Frauen nicht per se eine höhere Lebenserwartung haben und auch nicht zwangsläufig (genetisch) krankheitsanfälliger sind als Männer, sondern Gesundheit und Wohlbefinden im Alter von den Lebensumständen und der Biografie abhängen, sollen im Folgenden Kontextfaktoren von Gesundheit und Wohlbefinden im Alter in die geschlechterdifferenzielle Analyse einbezogen werden.

\section{Kontextfaktoren von Gesundheit und Wohlbefinden im Alter}

Im Unterschied zu biologischen Erklärungsansätzen für Unterschiede in Gesundheit und Wohlbefinden folgen wir epidemiologischen Sichtweisen, die dem (Risiko)Verhalten und den Aktivitätsmustern im Lebensverlauf eine bedeutende Rolle zusprechen (Baltes et al. 1994: 75). Sowohl bei kardiovaskulären Erkrankungen wie auch bei kognitiven Beeinträchtigungen (einschl. 
Demenz) und psychischen Krankheiten konnte nachgewiesen werden, dass zusätzlich zur genetischen Prädisposition krankheitsauslösende oder auch protektive Faktoren hinzukommen, die schlussendlich zur Erkrankung führen bzw. den Ausbruch der Krankheit verhindern. Zu den Risikofaktoren gehören der Konsum von Genussgiften und Vorerkrankungen, während ein hoher Bildungsstand, körperliche und geistige Aktivität sowie eine ausgewogene Ernährung protektiv wirken (vgl. Sattler/Schröder 2011). Als weitere Kontextfaktoren sind soziale Unterstïtzung und Netzwerke anzusehen. Thnen kann vor allem im Hinblick auf biografische Wendepunkte und die Bewältigung von Beeinträchtigungen im Lebenslauf eine große Bedeutung beigemessen werden, da Integration, Partizipation und Wertschätzung innerhalb der jeweiligen Netzwerke als bedeutsam für die Entwicklung von Handlungskompetenz i.S. von Resilienz anzusehen ist. Hieraus können Impulse für Lernprozesse entstehen, die über die unmittelbare Krisenbewältigung hinausgehen (vgl. Iller/Wienberg 2011).

Betrachtet man sich die Statistiken zur Zeitverwendung älterer Menschen, so zeigt sich, dass Frauen in stärkerem Maße Hausarbeit übernehmen und daher über weniger freie Zeit als Männer verfügen (vgl. Engstler 2004). Aktivitäten in Vereinen o.ä. wird in der nachberuflichen Phase überwiegend von Männern nachgegangen. Generell scheint das sportliche Aktivitätsniveau bei den befragten Männern höher als bei den Frauen zu liegen (vgl. Gärtner et al. 2005; zu konträren Ergebnissen vgl. Tippelt et al. 2009). Gemeinhin ist ein alterskorrelierter Rückgang der Beteiligung an allgemeiner und beruflicher Weiterbildung zu verzeichnen. Hierbei sind jedoch Geschlechterunterschiede zu erkennen: Bei Männern steigt die Weiterbildungsquote bis zum 60. Lebensjahr, danach ist ein Rückgang zu erkennen. Hingegen kommt es bei den Frauen zu einem Anstieg des non-formalen Lernens im Alter, außer bei den 55- bis 59-Jährigen (Kolland/Ahmadi 2010: 48). In den von uns durchgeführten qualitativen Interviewanalysen des ILSE-Datensatzes (Alterskohorte 1930 bis 1932) konnte festgestellt werden, dass Weiterbildungsaktive auch generell häufiger in informellen Kontexten lernen (vgl. Iller/ Wienberg 2011).

Im Hinblick auf die Struktur als auch die Qualität der sozialen Netzwerke konnten, u.a. in der European Study of Adult Well-Being (ESAW), Geschlechtsunterschiede sichtbar gemacht werden. Männer verfügen demnach zwar über mehr soziale Unterstïtzung als weibliche Befragte, Frauen verfïgen jedoch über mehr Kontakte und AnsprechpartnerInnen in persönlichen Dingen (vgl. Weber et al. 2005). Hinsichtlich der Zufriedenheit mit den sozialen Beziehungen bestehen laut den Ergebnissen der ESAW-Studie keine geschlechtsspezifischen Unterschiede (vgl. Weber et al. 2005). Insgesamt gibt es nur geringe Unterschiede zwischen älteren Männern und Frauen im Vorkommen und der zeitlichen Dauer informeller Hilfeleistungen, allerdings bestehen deutliche Geschlechtsunterschiede in den gegebenen Hilfearten. Handwerkliche Hilfe, Gartenarbeit, Transportaufgaben werden häufiger von 
Männern übernommen, während die Hilfe bei der Hausarbeit, dem Einkaufen und der Kinderbetreuung überwiegend durch die Frauen erbracht wird.

Unsere Analyse von ILSE-Daten ergab, dass strukturell gegebene familiäre wie auch außerfamiliäre Netzwerkstrukturen keinen Automatismus für Unterstützungsleistungen darstellen (vgl. Iller/Wienberg 2011). Bei der Analyse der Wirkungen von sozialen Beziehungen ist es deshalb unabdingbar, der Qualität sozialer Beziehungen eine hohe Bedeutung beizumessen. $\mathrm{Zu}$ der differenzierten Wahrnehmung der Beziehungsqualität gehört auch, die Rollenwechsel in Beziehungen zu berücksichtigen. Häufig verändern sich in familiären Beziehungen die „Unterstützungsverhältnisse“ zwischen den Generationen, aber auch intragenerational.

\section{Fazit und Ausblick}

Wie die dargestellten Forschungsergebnisse zeigen, ist eine geschlechterdifferenzielle Analyse in der Alternsforschung dringend erforderlich, denn sowohl hinsichtlich Gesundheit und Wohlbefinden als auch in den Kontextfaktoren lassen sich Geschlechterunterschiede erkennen. ${ }^{3}$ Zusammenfassend zeigen die Sekundäranalyse sowie eigene qualitative Untersuchungen, dass sich die Aktivitätsstruktur und soziale Vernetzung des jungen und mittleren Erwachsenenalters - wenn auch in einem anderen Ausmaß - im hohen Alter fortsetzen (,Matthäus-Effekt‘). Dies legt den Schluss nahe, dass Ungleichheit in den Lebenslagen von Männern und Frauen im hohen Alter fortbestehen werden, wenn nicht der Übergang ins höhere Alter zu einer reflexiven Auseinandersetzung mit der eigenen Lebensweise und in diesem Kontext auch mit den Restriktionen der Geschlechterrollen stattfindet.

Unsere bisherigen Untersuchungen weisen diesbezüglich jedoch auf ein komplexes Wechselverhältnis von gesundem Lebensstil, Bildung und sozialer Unterstützung als Voraussetzungen für zufriedenes und gesundes Altern: (Ehrenamtliche) Tätigkeit und die Ausübung von Freizeitaktivitäten münden in einer Veränderung familiärer und außerfamiliärer Netzwerke. Ein intaktes soziales Netzwerk wiederum kann wie ein Puffer gegenüber negativen Effekten wirken und so den Prozess der Entwicklung von Handlungskompetenz unterstützen. Vieles deutet darauf hin, dass zentrale Weichenstellungen für das Leben im hohen Alter an den Übergängen im sog. Dritten Lebensalter (z.B. Übergang in die Nacherwerbsphase und die postparentale Familienphase) vorgenommen werden. $\mathrm{Ob}$ und inwiefern diese Übergänge Anlass für ge-

3 Aufgrund dessen wurde aktuell erstmals eine umfassende Datenbank zu Geschlechterunterschieden in großen klinischen Fächern der Inneren Medizin erstellt (vgl. http:/bioinformatics. charite.de/gender) 
schlechterbezogene Aushandlungsprozesse geben, ist bislang noch unklar. Wichtig ist vor allem, nicht einen Automatismus zu unterstellen, sondern den Alternsprozess als Impuls für eine reflexive Auseinandersetzung mit der eigenen Lebenssituation aufzugreifen.

\section{Danksagung}

Diese Publikation basiert auf Analysen, die im Projekt „Perspectives of Ageing in the process of social and cultural change" vorgenommen wurden. Das Projekt wird im Rahmen des Marsilius-Kollegs, einem Center for Advanced Study an der Universität Heidelberg, durch die Exzellenzinitiative des Bundes und der Länder in Deutschland gefördert. Wir danken allen Projektbeteiligten für ihren Beitrag.

\section{Literatur}

Baltes, Paul B./Mittelstraß, Jürgen/Staudinger, Ursula M. (1994): Alter und Altern: Ein interdisziplinärer Studientext zur Gerontologie. Berlin: de Gruyter.

Baltes, Margret M./Horgras, Ann L./Klingenspor, Barbara/Freund, Alexandra/Carstensen, Laura L. (1996): Geschlechtsunterschiede in der Berliner Altersstudie. In: Mayer, K. U./ Baltes, P. B. (Hrsg.): Die Berliner Altersstudie. Berlin: Akademie Verlag, S. 597-622.

Betula - Aging, Memory and Dementia: Department of Psychology, Umeå University (2005). http://www.betula.su.se/en/index.html [Zugriff: 19.10.2009]

Clemens, Wolfgang (1997): Frauen zwischen Arbeit und Rente. Lebenslagen in später Erwerbstätigkeit und frühem Ruhestand. Opladen: Westdeutscher Verlag.

Engstler, Heribert/Menning, Sonja/Hoffmann, Elke/Tesch-Roemer, Clemens (2004): Die Zeitverwendung älterer Menschen. In: Statistisches Bundesamt (Hrsg.): Alltag in Deutschland - Analysen zur Zeitverwendung (Band 43 der Schriftenreihe Forum der Bundesstatistik). Stuttgart: Metzler Poeschel, S. 216-246.

Gärtner, Karla/Grünheid, Evelyn/Luy, Marc (Hrsg.) (2005): Lebensstile, Lebensphasen, Lebensqualität - Interdisziplinäre Analysen von Gesundheit und Sterblichkeit aus dem Lebenserwartungssurvey des BiB. Schriftreihe des Bundesinstituts für Bevölkerungsforschung, Band 36. Wiesbaden: Verlag für Sozialwissenschaften.

Iller, Carola (2005): Altern gestalten - berufliche Entwicklungsprozesse und Weiterbildung im Lebenslauf. http://www.die-bonn.de/esprid/dokumente/doc-2005/ iller05_12.pdf [Zugriff: 30.11.2011]

Iller, Carola/Wienberg, Jana (2010): ,Ältere“ als Zielgruppe in der Erwachsenenbildung oder Ansätze einer Bildung in der zweiten Lebenshälfte? In: Magazin Erwachsenenbildung.at - Das Fachmedium für Forschung, Praxis und Diskurs 10, S. 15-24. 
Iller, Carola/Wienberg, Jana (im Druck): Learning in social relationships - a Contribution to Successful Ageing? In: International Journal for Education and Aeging (IJEA).

Kickbusch, Ilona (2006): Gesundheitskompetenz. In: Public Health News 3-2006. http://www.gesundheitskompetenz.ch/request.php?site=definitionen\&siteID=112 \&lang=de [Zugriff: 20.12 .2009 ]

Kolland, Franz/Ahmadi, Pegah (2010): Stabilität und Wandel: Bildung im Lebenslauf. In: REPORT - Zeitschrift für Weiterbildungsforschung, 33(3), S. 43-53.

Kuhlmey, Adelheid/Mollenkopf, Heidrun/Wahl, Hans-Werner (2007): Gesund altern - ein lebenslauforientierter Entwurf. In: Wahl, H.-W./ Mollenkopf, H. (Hrsg.): Alternsforschung am Beginn des 21. Jahrhunderts. Alterns- und Lebenslaufkonzeptionen im deutschsprachigen Raum. Berlin: Akademische Verlagsgesellschaft, S. 265-274.

Lindenberger, Ulman /Smith, Jacqui/Mayer, Karl Ulrich/Baltes, Paul B. (Hrsg.) (2010): Die Berliner Altersstudie (3., erw. Aufl.). Berlin: Akademie Verlag.

Mayer, Karl Ulrich (1991): Berufliche Mobilität von Frauen in der Bundesrepublik Deutschland. In: Mayer, K.U./Allmendinger, J./Huinink, J. (Hrsg.): Vom Regen in die Traufe: Frauen in Beruf und Familie. Frankfurt a.M.: Campus, S. 57-90.

Perrig-Chiello, Pasqualina/Stähelin, Hannes B./Perrig, Walter J. (Hrsg.) (1999): Wohlbefinden, Gesundheit und kognitive Kompetenz im Alter. Ergebnisse der Basler Interdisziplinären Altersstudie IDA.

Sattler, C./Schröder, J. (im Druck): Kognitive Reserve im Alter - Risiko- und protektive Faktoren im Vorfeld der Alzheimer-Demenz. In: Schröder, J. \& Pohlmann, M. (Hrsg.): Gesund altern - individuelle und gesellschaftliche Herausforderungen. Heidelberg: Universitätsverlag Winter.

Schmitt, Marina/Wahl, Hans-Werner/Kruse, Andreas/Schröder, Johannes/Martin, Mike (2008): Konzeption, Leithypothesen und Ziele des dritten Messzeitpunkts der Interdisziplinären Längsschnittstudie des Erwachsenenalters (ILSE). Leithypothesen der ILSE und Schwerpunkte der Berichterstattung zum dritten Messzeitpunkt. In: Schmitt, M./Wahl, H.-W./Kruse, A. (Hrsg.): Interdisziplinäre Längsschnittstudie des Erwachsenenalters (ILSE). Abschlussbericht anlässlich der Fertigstellung des dritten Messzeitpunkts. Bonn: Bundesministerium für Familie, Senioren, Frauen und Jugend, S. 11-15.

Schröder, Johannes/Sattler, Chrsitine/Lamparter, Heidrun/Toro, Pablo (2008): Ergebnisse des dritten Messzeitpunkts und erste längsschnittliche Analysen. Befunde zur körperlichen und psychischen Gesundheit. In: Schmitt, M./Wahl, H.-W./ Kruse, A.: Interdisziplinäre Längsschnittstudie des Erwachsenenalters (ILSE). Abschlussbericht anlässlich der Fertigstellung des dritten Messzeitpunkts. Bonn: Bundesministerium für Familie, Senioren, Frauen und Jugend, S. 30-44.

Sørenson, Aage B. (1990): Gender and the life course. In: Kölner Zeitschrift für Soziologie und Sozialpsychologie, 42 (Sonderheft 32), S. 304-321.

Statistisches Bundesamt (2005): Schwerpunktbericht der Gesundheitsberichterstattung des Bundes. Gesundheit von Frauen und Männern im mittleren Lebensalter. Robert Koch-Institut, Berlin. http://edoc.rki.de/documents/rki_fv/ren4T3cctjHcA/ PDF/ 24KaxXc7rW0E_42.pdf [Zugriff: 07.10.2011]

Statistisches Bundesamt (2008a): Basisdaten Lebenserwartung von Frauen bei Geburt. http://www.destatis.de/jetspeed/portal/cms/Sites/destatis/Internet/DE/Content/Sta 
tistiken/Internationales/InternationaleStatistik/Thema/Tabellen/Basistabelle Le benserwartungw.psml [Zugriff: 13.04.2011]

Statistisches Bundesamt (2008b): Basisdaten Lebenserwartung von Männern bei Geburt. http://www.destatis.de/jetspeed/portal/cms/Sites/destatis/Internet/DE/Content/ Statistiken/Internationales/InternationaleStatistik/Thema/Tabellen/Basistabelle Lebenserwartungm.psml [Zugriff: 13.04.2011]

Statistisches Bundesamt (2009): Ergebnisse der Todesursachenstatistik für Deutschland - Ausführliche vierstellige ICD 10-Klassifikation. http://www.destatis.de/ jetspeed/portal/cms/Sites/destatis/Internet/DE/Content/Publikationen/Fachveroeff entlichungen/Gesundheit/Todesursachen/Todesursachenstatistik,templateId=rend erPrint.psml [Zugriff: 10.10.2011]

Tesch-Römer, Clemens/Wurm, Susanne (2009): Theoretische Positionen zu Gesundheit und Alter. In: Böhm, K./Tesch-Römer, C./Ziese, T. (Hrsg.): Beiträge zur Gesundheitsberichterstattung des Bundes. Gesundheit und Krankheit im Alter. Berlin, S. 7-20.

Tews, Hans Peter (1993): Neue und alte Aspekte des Strukturwandels des Alters. In: Naegele, G./Tews, H. P (Hrsg.): Lebenslagen im Strukturwandel des Alters. Opladen: Westdeutscher Verlag, S. 15-42.

Tews, Hans Peter (1999): Von der Pyramide zum Pilz. Demographische Veränderungen in der Gesellschaft. In: Niederfranke, A. Naegele, G./Frahm, E. (Hrsg.): Funkkolleg Altern 1. Die vielen Gesichter des Alterns. Opladen/Wiesbaden: Westdeutscher Verlag, S. 137-185.

Tippelt, Rudolf/Schmidt, Bernhard/Schnurr, Simone /Sinner, Simone /Theisen, Catharina (2009): Bildung Älterer - Chancen im demografischen Wandel, DIE-Spezial. Bielefeld: Bertelsmann Verlag.

Weber, Germain/Glück, Judith/Heiss, Cecilia/Sassenrath, Simone/Schäfer, Lars/ Wehinger, Katharina (2005): ESAW - European Study of Adult Well-Being (20022004): Hauptergebnisse unter besonderer Berücksichtigung der Situation in Österreich. Wien: Facultas Verlag.

WHO MONICA Projekt (2009). http://www.ktl.fi/monica/ [Zugriff: 12.04.2011]

Wienberg, Jana (2010): Eine Feldsondierung zum Thema Bildung und Gesundheit im Alter. In: Der Pädagogische Blick, 1, S. 37-49. 